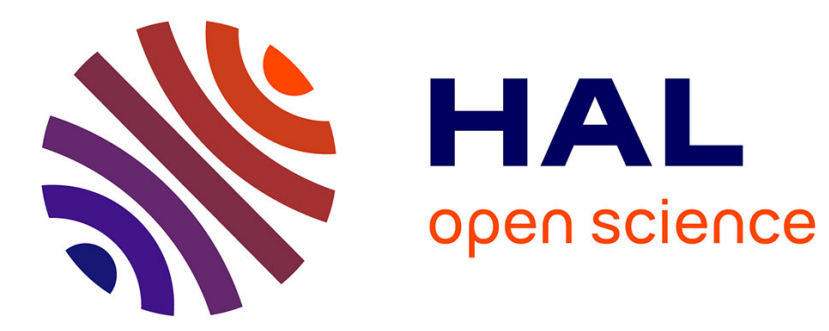

\title{
L'empan liminal, un outil conceptuel pour penser la scolarisation des élèves de Clis 1
}

Claire de Saint Martin

\section{To cite this version:}

Claire de Saint Martin. L'empan liminal, un outil conceptuel pour penser la scolarisation des élèves de Clis 1. La nouvelle revue de l'adaptation et de la scolarisation, 2016, 73 (1), pp.239-251. 10.3917/nras.073.0239 . hal-03163319

\section{HAL Id: hal-03163319 \\ https://hal.science/hal-03163319}

Submitted on 9 Mar 2021

HAL is a multi-disciplinary open access archive for the deposit and dissemination of scientific research documents, whether they are published or not. The documents may come from teaching and research institutions in France or abroad, or from public or private research centers.
L'archive ouverte pluridisciplinaire HAL, est destinée au dépôt et à la diffusion de documents scientifiques de niveau recherche, publiés ou non, émanant des établissements d'enseignement et de recherche français ou étrangers, des laboratoires publics ou privés. 


\title{
L'empan liminal, un outil conceptuel pour penser la scolarisation des élèves de Clis $1^{*}$
}

\author{
Claire DE SAINT MARTIN \\ Docteure en sciences de l'éducation \\ Ater en sciences de l'éducation \\ Laboratoire Éducation, mutations, apprentissages \\ Espé - Université de Cergy-Pontoise, France
}

Résumé: À l'école élémentaire, les Classes pour l'inclusion scolaire de type 1 (Clis 1) sont des dispositifs adaptés accueillant des élèves connaissant des troubles des fonctions cognitives ou mentales, ou des troubles envahissants du développement. Ces classes constituent le lieu de la liminalité, telle que l'a défini Murphy, à savoir un entre-deux qui place les personnes handicapées aux marges de la société. Que peuvent alors dire ces élèves de leur place dans l'école? Une recherche collaborative menée avec les élèves de trois Clis 1 a permis d'étudier leur situation liminale par l'organisation de séances de réflexion collective, complétées par des temps d'observation participante. La liminalité se définit ici comme un processus dynamique. La conceptualisation de l'empan liminal, révélant différentes dimensions, individuelle, structurelle, sociale, culturelle, permet de penser la scolarisation de ces élèves et de questionner l'éducation inclusive.

Mots-clés: Clis 1 - Empan liminal - Handicap - Inclusion - Liminalité - Recherche collaborative - Socio-clinique institutionnelle - Sociologie de l'enfance.

The liminal span, a conceptual tool for thinking the schooling of pupils in a specialized class called Clis 1

Summary: In elementary school, the Classes for school inclusion type 1 (Clis 1) are adapted settings with pupils having disabilities affecting cognitive or mental functions, or pervasive developmental disorders. These classes can be considered as the place of liminality, such as defined by Murphy, an in-between that places people with disabilities at the margin of society. What can those pupils say about their place at school ? We can study their liminal status through collaborative research with pupils from three Clis 1 by organizing brainstorming sessions complemented by participant observation time. Liminality can be defined here as a dynamic process. The conceptualization of the liminal span, revealing different dimensions, individual, structural, social, cultural, allows to think about the schooling of these children and to question the inclusive education.

Keywords: Clis 1 - Collaborative research - Disability - Inclusion - Institutional socio-clinic - Liminal span Liminality - Sociology of childhood.

\footnotetext{
* Les Classes pour l'inclusion scolaire (Clis) ont été créées par la circulaire 2009-087 du 17 juillet 2009 et sont définies comme un " dispositif collectif de scolarisation ". Elles ont succédé aux Classes d'intégration scolaire (Clis), initialement mises en place par la circulaire 91-304 du 18 novembre 1991. Une circulaire récente en date du 21 août 2015 (n²015-129) transforme les Classes pour l'inclusion scolaire en "Unités localisées pour l'inclusion scolaire-école (Ulis école) " et les définit comme " un dispositif qui offre aux élèves qui en bénéficient une organisation pédagogique adaptée à leurs besoins ainsi que des enseignements adaptés dans le cadre de regroupement " (1.2).
} 

différentes dimensions, institutionnelle, culturelle, sociale, individuelle. Ce concept enrichit celui de liminalité, tel que l'a pensé Murphy à partir des travaux anthropologiques de Van Gennep (2001) et de Turner (1990). Devenu tétraplégique, Murphy théorise la situation des personnes avec handicap moteur par la liminalité, qui les place aux marges de la société. Leur handicap les empêche d'y participer pleinement pour des raisons sociales: les valides, sans les rejeter complètement, ne les acceptent pas non plus pleinement. Au contraire des rites liminaires décrits par Van Gennep et Turner, la liminalité définit ici une situation irréversible et dramatique. Les individus handicapés sont définitivement maintenus dans la phase liminaire parce que leur écart à la forme ordinaire représente une menace pour l'ordre social, du fait d'une impossibilité d'expérience commune, du déni par la société de leur "être de culture" (Murphy, 1990 : 75). La liminalité a plusieurs effets: structurels par une place différente accordée à la personne dans la société ; culturels par la transformation de leurs relations avec les valides; individuels par le sentiment de honte de l'individu handicapé.

Des chercheurs français ont repris le concept de Murphy pour caractériser toute forme de handicap (Calvez, 1994; Gardou, 2005; Sticker, 2005; Blanc, 2006). Si Blanc reconnaît une origine endogène au handicap, les autres chercheurs le reconnaissent comme un fait exclusivement culturel, sociologique, volontaire et conscient, concernant tous les acteurs de la société et utilisé par tous. "La liminalité désigne cette situation de seuil dans laquelle l'individu flotte dans les interstices de la structure sociale. " (Calvez, 1994 : 61).

Au sein de l'école élémentaire, les Clis sont des classes spécialisées qui accueillent un maximum de douze élèves en situation de handicap. Les Clis 1 accueillent spécifiquement des élèves "dont la situation de handicap procède de troubles cognitifs ou mentaux. En font partie les troubles envahissants du développement ainsi que les troubles spécifiques du langage et de la parole " (circulaire $n^{\circ} 2009-087$ du 17 juillet 2009). Ces élèves passent plusieurs années dans la même classe, au contraire de ceux de l'ordinaire. Ils fréquentent donc un nombre restreint de pairs pendant leur scolarité. Ils doivent cependant connaître des temps de scolarisation en classe ordinaire, définis par les enseignants selon la problématique de chacun. La Clis 1 est le lieu de la liminalité. Les élèves qui y sont accueillis ne sont pas exclus de la société : ils ne sont pas placés en établissement spécialisé. Mais ils ne sont pas non plus inclus entièrement dans l'école, puisqu'ils sont dans une classe spécialisée. Que peuvent alors dire ces élèves de leur place dans l'école? Pour questionner le concept de liminalité, j'ai créé, dans trois Clis différentes de la région parisienne, nommées Balzac, Baudelaire et Diderot, un dispositif de réflexion collective auprès des élèves.

Après avoir présenté la recherche collaborative, j'expliciterai le concept d'empan liminal et envisagerai ensuite en quoi ce concept enrichit celui de Murphy.

\section{UNE RECHERCHE COLLABORATIVE}

L'attention aux discours des élèves de Clis 1 permet de rendre compte de leurs perceptions de leur situation de liminalité au regard de cette socialisation particulière 
qui les isole de cette population scolaire tout en les inscrivant dans l'école. La recherche collaborative s'est construite à l'appui des cadres théoriques et méthodologiques de la socio-clinique institutionnelle et de la sociologie de l'enfance.

\section{Chercher avec les acteurs de terrain}

Avec la collaboration des enseignantes, j'ai organisé des séances de réflexion collective auprès des élèves, à partir de la question de leur place dans l'école.

La recherche collaborative s'est déroulée sur six mois. De novembre à décembre 2012, j'ai rencontré les enseignantes des trois Clis, Fabienne, Amélie et Marine, et nous avons organisé le dispositif. Entre janvier et avril 2013, j'ai rencontré dix fois les élèves, à raison d'une séance hebdomadaire dans chaque classe. En raison des temps de scolarisation différenciés, vingt-neuf élèves sur trente-six ont participé à ces temps de réflexion collective. Pour introduire le concept de liminalité, j'ai explicité le projet en leur disant que j'avais observé qu'ils étaient les seuls élèves de l'école à aller dans plusieurs classes. Je leur proposais donc de réfléchir collectivement à leur place dans l'école. Les élèves ont d'abord pris des photos individuelles des places de la classe et de l'école qu'ils préfèrent et qu'ils détestent. Ces photos présentées, explicitées et commentées en groupe, ont révélé des constantes qui ont servi de point de départ à la réflexion collective, enregistrée. L'introduction d'un bâton de parole a régulé les échanges chaotiques de la première séance. II signalait visuellement celui qui parlait. II a permis à chacun de prendre le temps d'élaborer sa pensée avant de la verbaliser et d'instaurer une véritable écoute, faisant émerger dans chaque classe la réflexion collective. Quels que soient la formulation des questions et les cheminements réflexifs, toutes les classes ont traité des mêmes thèmes: la question des difficultés, les différences entre la Clis et les classes d'inclusion, le fait d'avoir plusieurs places dans l'école, leurs relations entre eux, les relations avec les adultes et avec les autres élèves de l'école, les intervenants extérieurs. Dans deux Clis, la dernière séance a été consacrée à la réalisation d'un écrit collectif, sous la forme d'une exposition adressée aux parents dans l'une (légender les photos à partir de la réflexion collective), sous la forme d'une lettre ouverte aux élèves de l'école dans l'autre.

Les enseignantes ont choisi le moment de mon intervention, sélectionnant de fait les élèves qui y participaient, puisque certains étaient accueillis à temps partiel dans l'école, d'autres étaient en classe ordinaire au moment de mon intervention. Nous avons construit ensemble le dispositif, constamment réévalué en fonction du déroulement des séances: les enseignantes assistaient aux séances sans diriger la réflexion. Celles des Clis Balzac et Diderot ont demandé à faire une séance en demi-groupe, pour que les plus jeunes élèves participent davantage, sans être systématiquement repris par les plus grands. Cette séance a effectivement libéré la parole de certains enfants. Nous nous sommes aussi adaptées aux demandes des élèves. Ce sont ceux de la Clis Baudelaire qui ont décidé la forme de l'écrit final, une lettre ouverte aux autres acteurs de l'école.

Les élèves ont élaboré la réflexion collective en autonomie dans la mesure où je leur servais seulement de guide dans celle-ci. Mais c'est moi qui déterminais les thèmes abordés en fonction de leurs discours, des intérêts qu'ils manifestaient et 
de la confrontation de ces discours à mes observations en dehors de ce temps réflexif. Les élèves se sont progressivement approprié le dispositif, échangeant véritablement entre eux. L'évolution de mes interventions a marqué celle de la réflexion: nombreuses lors des premières rencontres pour activer leur parole, j'ai pu les réduire au cours des séances. La nécessité de relancer plus souvent les élèves à partir de la sixième séance a été l'indice de l'épuisement du sujet.

\section{La socio-clinique institutionnelle}

Toute démarche socio-clinique nécessite une pluralité de données pour activer la production de connaissances en situation (Fablet, 2004). En participant à la vie de l'institution étudiée, le chercheur puise dans l'ethnographie son travail d'observation. Cette démarche qualitative ne peut pas se suffire d'entretiens, individuels ou collectifs. Je restais la demi-journée dans chaque classe. Les séances, qui duraient une quarantaine de minutes, étaient complétées par une observation participante dans la cour de récréation, à la cantine et durant les activités de classe. J'ai conclu ces séances par des entretiens individuels avec les enfants volontaires et deux AVS ${ }^{1}$ sur trois. Un bilan final a été réalisé avec chaque enseignante, bilan renouvelé au mois de juin, afin d'évaluer à long terme les effets du travail mené avec les élèves, ces effets apportant des données complémentaires aux autres résultats. En proposant aux élèves de Clis 1 de réfléchir sur leur place dans l'école, je les amenais à élucider leurs propres rapports à l'institution scolaire.

La socio-clinique institutionnelle a été théorisée par Monceau (2003) dans le cadre de l'analyse institutionnelle (Lourau, 1970). Elle propose aux acteurs de terrain de réfléchir sur leurs pratiques dans une relation de proximité avec le chercheur qui se trouve ainsi impliqué, au sens de l'analyse institutionnelle, c'est-à-dire pris dans l'institution qu'il étudie. La présence même du chercheur dérange celle-ci. Cette perturbation est méthodologiquement produite. "De manière plus générale, la relation est interaction, action de chacun des actants sur les autres. Elle est interférence puisque produisant, de plus, autre chose que la transformation de chacune des deux parties. " (Monceau, 2005). Le chercheur ne pose pas d'hypothèses au préalable mais analyse les effets produits par son intervention, effets imprévisibles ou différents de ceux attendus. Par la production d'une analyse collective en situation conduisant à un changement des pratiques, la socio-clinique institutionnelle comporte une part d'incertitude que le chercheur accepte et assume.

Chercher ainsi avec les acteurs de terrain oblige à une analyse des effets qui se produisent au cours de la recherche. Dans ce contexte, l'analyse des implications, le travail des analyseurs et les restitutions constituent des éléments centraux de la recherche. Les implications sont entendues comme les "rapports organisationnels, idéologiques et libidinaux " (Monceau, 2012: 7) de chaque individu à l'institution. Leur analyse collective permet d'étudier les différents rapports de force en analysant les pratiques professionnelles. Le travail des analyseurs, à savoir tout événement surgissant au cours de l'analyse collective et obligeant chacun à prendre position,

1. AVS: Auxiliaire de vie scolaire, secondant l'enseignant dans la classe et pouvant aider les élèves dans tous les moments de la vie scolaire (cantine, récréation, temps périscolaires). 
met en évidence les contradictions de l'institution et montre comment le macro est contenu dans le micro, révélant la continuité entre les pratiques et les décisions institutionnelles à tout niveau. Les analyseurs sont explicités au cours des restitutions aux acteurs de terrain. J'en ai mené de deux types: au cours de la réflexion collective, je faisais des restitutions perlées (Monceau, $2003: 30$ ), synthèses de ce qui venait d'être dit, vérifiant la solidité des discours et relançant la réflexion. À la fin de chaque séance, la discussion informelle avec l'enseignante, où chacune en donnait son interprétation, constituait également une restitution perlée. Chaque séance faisait l'objet d'une synthèse écrite par mes soins reprenant exclusivement les propos des élèves. Lue au début de la réflexion collective suivante, elle était validée ou corrigée collectivement. Ces différents éléments menaient à une adaptation permanente du dispositif, au fil de la progression de la réflexion collective.

Ces adaptations réfléchies avec tous les acteurs de la recherche, dans une écoute et un respect communs, ont permis à chacun d'intervenir à sa mesure, de maintenir l'intérêt des enfants pour le projet, et de faire émerger une véritable réflexion. Les choix opérés et les réajustements successifs ont tenu compte des réalités spécifiques de chaque classe, mais aussi d'un souci éthique constant d'assurer aux enfants la considération de leurs discours et l'absence de tout jugement. C'est bien cette position éthique, comprise par les enfants, qui a libéré leur parole et autorisé l'émergence de positions parfois inavouables dans le cadre scolaire.

\section{La sociologie de l'enfance}

La sociologie de l'enfance permet de saisir l'enfant " comme un être humain au présent et non pas seulement en devenir " (Kay, Tisdall, Davis et Callagher, 2009 : 2). L'enfant est reconnu comme un acteur social, individuellement et collectivement, au sein d'un groupe de pairs. Il participe activement à la construction de sa socialisation, peut rapporter lui-même son expérience et sa vision du monde. II s'agit alors de comprendre " ce que l'enfant fait de ce qu'on lui fait » (Sirota, 2006 : 21).

Mais comment faire participer des enfants reconnus déficients intellectuels pour qui la pensée, la parole ne vont pas toujours de soi? Par leur inscription en Clis 1, les élèves sont considérés dans l'espace scolaire prioritairement, sinon exclusivement, sous le prisme du handicap. Ce sont leurs défaillances et leurs besoins qui déterminent les conditions de leur scolarisation. Ils sont d'abord envisagés par ce qu'ils ne peuvent pas faire. Or, selon Freire (2013), tout individu, même le plus faible, est capable d'apprendre. Considérer d'abord les élèves de Clis 1 par leurs potentialités change le regard porté sur eux et autorise des possibles impensables sans cette perspective. C'est le parti-pris méthodologique et épistémologique que j'ai assumé. Je n'en ai pas pour autant nié leurs difficultés, mais les ai considérées dans leur fonctionnalité en m'adaptant aux situations rencontrées: faire participer une élève non verbale en lui posant des questions fermées auxquelles elle répondait gestuellement; laisser les enfants, habitués au langage incompréhensible d'un des leurs traduire ses propos... Restait la question du handicap. Les élèves de Clis 1 sont identifiés par les acteurs de l'école sous cette seule étiquette qui produit des effets sociaux. Les sens savant et institutionnel du mot, qui reconnaissent la responsabilité de la société, ne parviennent pas à s'imposer face aux représentations communes. Certains parents 
refusent l'admission en Clis 1, parce qu'elle se fait par la Maison départementale des personnes handicapées, alors qu'ils dénient un handicap à leur enfant, cette dénomination réduisant son identité à cette étiquette dévalorisante. Je devais tenir compte de cette représentation sociale qui risquait de voir les élèves interpréter cette approche comme une dévalorisation d'eux-mêmes, et cela d'autant plus que tous ne relèvent pas du champ du handicap, comme je le développerai plus loin. J'ai donc décidé de ne pas les aborder par le handicap et de ne pas prononcer le mot, laissant ainsi aux élèves le choix de l'introduire.

La recherche ainsi menée a permis de mettre au jour le concept d'empan liminal.

\section{L'EMPAN LIMINAL}

Tous les élèves reconnaissent leurs difficultés qui justifient leur orientation en Clis.

Clis Baudelaire, $1^{\text {re }}$ séance de réflexion, lundi 4 février 2013

Tom: "Moi, en CP et en CE1, j'ai eu des difficultés et donc je suis venu en Clis."

Éric: "Moi, j'avais des difficultés aussi et je suis venu en Clis. "

Alec: " J'étais au CP, j'arrivais pas à faire les additions des plus et je faisais

que des jeux, que des coloriages. J'arrivais pas à faire... le travail. "

Leurs perceptions de leur place dans l'école diffèrent cependant selon leurs temps de scolarisation en classe ordinaire et selon le nombre d'années passé en Clis. Ces différences permettent de parler d'un empan liminal et de considérer ici la liminalité comme un processus dynamique.

\section{Des perceptions différentes selon les situations scolaires}

Une opposition forte se marque entre les élèves qui connaissent de vrais temps de scolarisation en classe ordinaire et ceux qui n'y vont qu'en raison de l'absence de la maîtresse. Ces derniers ont une représentation faussée des activités des classes ordinaires, parce qu'ils n'y connaissent pas de temps d'apprentissage.

Clis Balzac, $6^{\mathrm{e}}$ séance de réflexion collective, mardi 19 mars 2013

Stéfy: "Moi, je me plais dans une autre classe, aller chez Mme P... Je joue à des jeux. Elle me laisse jouer. "

Et si tu y allais tout le temps, tu penses que tu jouerais tout le temps?

Stéfy: "Oui. "

Ces élèves n'ont pas de preuves à donner quand ils vont dans une autre classe à l'occasion de l'absence de leur maîtresse. Ces temps sont considérés comme des temps ludiques qui leur plaisent parce qu'ils sont déchargés de tout enjeu scolaire. Ces élèves se satisfont de leur place dans l'école et n'en revendiquent pas d'autre.

Clis Balzac, présentation et justification des photos, mardi 22 janvier 2013

Stéfy: " J'ai pas d'inclusion." "

Est-ce que tu aimerais en avoir?

Elle fait signe que non.

Tu es bien dans la classe de Clis?

Elle fait signe que oui. "La Clis, ça me donne du bonheur."

Certains élèves connaissent en classe ordinaire des temps de scolarisation très limités, une fois par semaine, qui ne concernent pas les apprentissages fondamentaux mais des activités sportives, artistiques ou de lecture d'album. De ce fait, leurs 
perceptions des apprentissages dans les classes ordinaires restent parcellaires. Dès lors, certains expriment leur envie d'y aller pour des activités qui ne correspondent pas à des temps d'apprentissage. "Oui. Moi, je voudrais travailler dans une autre classe, parce que j'aime bien faire des coloriages, du dessin. " (Aurore, Clis Balzac) Ils se satisfont cependant de cette situation dans l'école et ne désirent majoritairement pas en changer.

Clis Baudelaire, 5e séance de réflexion collective, lundi 18 mars 2013

Riad: "Moi pas d'accord, La CLI aidé les gens."

La Clis est faite pour aider?

Riad: "Oui. Les gens. Très bien."

Toi, tu es très content d'être en Clis?

Riad: "Oui. "

Certains élèves vont en classe ordinaire pour des apprentissages fondamentaux, toujours en CP ou en CE1. Ils sont souvent plus âgés que les élèves de ces classes de deux ou trois ans, voire plus. La situation liminale est plus difficile à vivre pour eux, parce qu'ils rencontrent de grandes difficultés d'apprentissage dans la classe ordinaire. "En fait, j'aime pas trop les CPA, j'aime plus ma classe, parce qu'on fait plus de choses, que la maitresse m'aide. II y a des choses dans le travail de CPA, j'arrive pas, j'aime pas trop bien. La maîtresse me donne du travail et il y a des choses que je ne comprends pas trop bien. " (Aziza, Clis Diderot). Ces difficultés, la différence d'âge exacerbent leurs différences et la conscience de leur écart avec la norme scolaire. Même s'ils revendiquent la légitimité de leur présence à l'école, ces élèves sont ceux qui expriment le plus de plaintes et de revendications.

D'autres élèves font des allers retours quotidiens entre la Clis et une ou plusieurs classes ordinaires pour les apprentissages fondamentaux. "Pour moi, c'est un avantage, parce que j'aime bien aller dans deux classes, parce que là-bas, ça me donne à être fort en maths; et ici, ça me fait reposer et des fois faire le travail. " (Loman, Clis Balzac). La Clis soulage les élèves des efforts que représentent les apprentissages en classe ordinaire. Changer constamment de classe est cependant source de frustration. "Quand on a fait un gâteau, et ben moi et Loman... Loman, il a dit "eh ben moi, je peux pas finir le gâteau, parce que je vais partir dans la classe." " (Dany, Clis Balzac). Les changements constants, au cours d'une demi-journée souvent, obligent ces élèves en grande difficulté à des adaptations constantes qui ne facilitent pas l'entrée dans les apprentissages. Ils n'ont jamais le temps de se poser. Ces élèves ont une grande conscience des enjeux de la scolarisation en classe ordinaire.

\section{Différentes situations liminales}

Les temps de scolarisation en classe ordinaire adaptés à chaque enfant permettent de définir différentes situations liminales, déclinées dans un empan qui joue de plusieurs façons.

Quand l'élève reste en Clis ou ne connaît qu'un temps de scolarisation en classe ordinaire limité (une ou deux heures par semaine), il est satisfait de sa place dans l'école. Alors qu'il est placé dans une situation liminale maximale, il n'en a pas conscience, parce que le fait d'avoir une seule place dans l'école lui permet de se considérer comme un élève au même titre que les autres. Le travail en Clis étant 
adapté aux besoins et aux rythmes spécifiques de chacun, ces élèves ont moins conscience de leurs difficultés d'apprentissage et considèrent uniquement leurs progrès. Cette situation liminale est moins éprouvante pour eux, parce qu'ils peuvent I'ignorer ou l'oublier. De plus, arrivés en Clis depuis au plus un an, ils n'éprouvent pas le besoin d'engager des relations avec d'autres élèves, se satisfaisant des liens créés avec leurs pairs dans la classe. Ils ne se perçoivent donc pas comme différents. Les élèves qui vivent une situation liminale médium, c'est-à-dire qui connaissent des temps de scolarisation en classe ordinaire limités dans des domaines fondamentaux, sont ceux qui pointent davantage leurs différences avec les autres élèves de l'école, parce que leurs difficultés les exacerbent et qu'ils ne peuvent alors ni les ignorer, ni les oublier. Ce sont eux qui vivent le plus difficilement cette situation parce qu'ils sont confrontés à des normes scolaires auxquelles ils ne peuvent pas répondre et parce que leur fréquentation parcellaire d'élèves plus jeunes qu'eux ne leur permet pas d'engager des relations sociales continues et amicales. Celles-ci sont rares et majoritairement conflictuelles.

Enfin, les élèves qui vont quotidiennement et sur des temps conséquents en classe ordinaire connaissent une situation liminale minimale. Ils en conçoivent de la fierté et considèrent ces temps comme une récompense de leurs progrès scolaires. S'ils dénoncent les allers-retours permanents entre plusieurs classes, ils se montrent soulagés de pouvoir revenir en Clis pour se libérer de la pression scolaire ordinaire. Ce sont les seuls élèves qui se reconnaissent des copains à l'extérieur de la Clis, même si les observations faites dans la cour de récréation et à la cantine contredisent cette affirmation. De leur point de vue, le processus de normalisation est donc opérant à la fois sur le plan des apprentissages et sur celui de la socialisation.

La liminalité des élèves de Clis 1 se décline donc dans un empan, qui va d'une situation liminale maximale, quand l'élève reste en Clis, à une situation minimale, où l'élève intègre les injonctions parentale et scolaire de normalisation et s'efforce d'y répondre. Contrairement à la situation décriée par Murphy, la liminalité ici ne s'inscrit pas dans une réalité figée, mais dans un mouvement permanent, un processus dynamique qui autorise les élèves à valider leur place dans l'école.

\section{L'empan liminal, un processus dynamique}

L'élève scolarisé en Clis y reste plusieurs années parfois toute sa scolarité élémentaire. Les élèves qui ont participé à cette recherche sont en moyenne arrivés en Clis à l'âge de sept ou huit ans. La majorité y reste jusqu'à l'âge limite de scolarisation en élémentaire, à savoir douze ans. Cette scolarisation n'est cependant pas figée, parce qu'au cours des années, l'élève connaît progressivement des temps de scolarisation plus importants en classe ordinaire. Celui qui ne peut pas répondre à cette progression est voué à une orientation en établissement spécialisé, généralement un IME². L'impossibilité de l'intégrer en classe ordinaire est alors analysée comme la preuve de son inadaptation au système scolaire.

2. IME: Institut médico-éducatif, établissement spécialisé accueillant des enfants porteurs de déficiences intellectuelles. 
Cette évolution est identifiée par les élèves comme un effet direct de l'aide apportée par l'enseignante de Clis, qui leur permet de surmonter leurs difficultés rencontrées en classe ordinaire. L'augmentation du temps de scolarisation en classe ordinaire est perçue comme une reconnaissance de leurs progrès et une récompense de leurs efforts.

Clis Diderot, $2^{\mathrm{e}}$ séance de réflexion, vendredi 15 février 2013

Marine (l'enseignante): "Pourquoi tu vas dans d'autres classes pour travailler? "

Abdel: "Je travaille bien."

Le fait d'avoir plusieurs classes, c'est le signe que tu travailles bien?

Abdel: "Oui, en mathématiques, pour apprendre. "

Cette double scolarisation amène alors les élèves à manifester une distance par rapport aux autres élèves de la Clis, et à revendiquer leurs différences par rapport à eux.

Clis Baudelaire, $3^{e}$ séance de réflexion, lundi 18 février 2013

Éric: "Ce qui me gêne, c'est de venir [en Clis]. J'aime pas. "

Est-ce que tu peux expliquer ce qui te gêne dans le fait de venir?

Éric: " J'aime, mais... C'est pas une classe normale. "

[...]

Tom: "Parce que il y a des têtes, elles sont bizarres. "

Ainsi, l'augmentation des temps de scolarisation en classe ordinaire inscrit l'élève dans un processus de normalisation, qu'il revendique au fil de ces années en Clis, par une distanciation progressive de cette classe et une constatation objective de ses progrès scolaires.

La liminalité révèle aussi son caractère dynamique par les différents éprouvés individuels. Elle n'inscrit pas l'enfant à la place figée dénoncée par R. F. Murphy, mais lui permet de vivre différentes situations liminales, au fil de ses progrès scolaires.

\section{UN ENRICHISSEMENT DU CONCEPT DE MURPHY}

L'empan liminal met au jour une liminalité plurielle qui s'exprime dans différentes dimensions, individuelle, institutionnelle, structurelle, sociale et culturelle. Les discours des élèves confrontés aux observations ethnographiques révèlent également une liminalité évolutive qui possède un caractère bénéfique parce qu'elle permet leur maintien à l'école. Leur situation liminale n'en reste pas moins majoritairement pérenne.

\section{Une liminalité plurielle}

L'inscription en Clis institutionnalise l'entrée de l'élève dans le champ du handicap et ce d'autant plus qu'elle accueille des enfants qui ne relèvent pas toujours de ce champ. Dans la Clis Balzac, Fabienne, I'enseignante, dénonce cette orientation faite pour garantir une scolarité régulière à deux enfants du voyage par la prise en charge d'un transport. De même, Amélie, I'enseignante de la Clis Baudelaire, justifie la présence dans sa classe de Charlène par la maltraitance soupçonnée des parents. Le dispositif devient alors l'instrument du contrôle social au nom de la protection de l'enfant, perpétuant ainsi la tradition historique des classes spécialisées. C'est majoritairement l'école qui propose l'orientation en Clis, en raison de résultats scolaires insuffisants. Mais ceux-ci définissent-il de véritables troubles des fonctions cognitives ou des difficultés d'apprentissage? 
La scolarisation de ces élèves dans un dispositif spécialisé au sein de l'école élémentaire marque la liminalité structurelle. S'ils sont maintenus dans le système éducatif ordinaire, leur maintien en Clis les écarte des autres acteurs de l'école malgré les temps de scolarisation en classe ordinaire. La liminalité structurelle s'exprime spatialement, par des allers retours incessants entre plusieurs classes. Un élève de la Clis Diderot, âgé de dix ans fréquente ainsi quatre classes: il va en CE1 en mathématiques, en CE2 en sciences, en CM1 en EPS. Ces différentes places géographiques déterminent alors la dimension sociale de la liminalité.

Écartés du fonctionnement et de l'organisation ordinaires de l'école, les élèves de Clis 1 n'entretiennent avec les autres acteurs de l'école que des relations épisodiques, temporaires. Ils développent alors une sociabilité forte à l'intérieur même de la Clis. II s'agit d'une socialisation " structurelle ", parce que "leurs affinités dépassent alors le jeu, elles sont le résultat d'une vie commune" (Delalande, 2001 : 91). Mais cette socialisation les écarte du reste de la population scolaire pour laquelle ils ne sont alors plus identifiés que par le handicap. Dans l'école Diderot, des enfants venus se plaindre de Fabien et Sam auprès de Marine l'enseignante les nomment « les Clis ». Ces élèves ne peuvent pas véritablement connaître ceux de la Clis qu'ils ne différencient pas. Leurs relations sont dominées par les représentations sociales du handicap mental, toujours dévalorisantes.

La liminalité a alors des effets culturels. Les élèves de Clis 1 dénoncent la stigmatisation dont ils disent faire l'objet de la part des autres enfants. Les élèves de la Clis Diderot la justifient par l'emploi du mot handicapé comme une insulte.

Clis Diderot, $2^{\mathrm{e}}$ séance de réflexion collective, vendredi 15 février 2013

Et comment ça se fait que vous soyez en Clis alors?

Fabien: "Parce que on a des difficultés, on a des problèmes. "

Didier: "Non, je suis pas handicapé."

Abdel: "On n'est pas handicapés. On a un problème de langage. » [...]

Didier: "Tout le monde dit qu'on est handicapés. "

On retrouve ici l'effet culturel de la liminalité décrite par Murphy: la transformation des relations par le regard négatif des valides sur les personnes en situation de handicap. Cependant, ce regard n'est pas ici toujours avéré. Les élèves de la Clis Balzac ne se plaignent pas des autres. Mais, dans cette classe, l'enseignante ne scolarise en classe ordinaire que les élèves pouvant suivre le programme scolaire de leur âge, à un ou deux ans près. De ce fait, huit enfants sur douze restent à plein temps en Clis et ne fréquentent pas les autres élèves de l'école. Marine, l'enseignante de la Clis Diderot précise qu'une seule élève a insulté les siens en les traitant de handicapés. Mais ce fait a suffisamment marqué les enfants pour qu'ils y reviennent constamment et justifient leur méfiance à l'égard de tous les autres élèves. Le même sentiment conduit les élèves de la Clis Baudelaire à écrire une lettre ouverte à l'ensemble des acteurs de l'école.

Clis Baudelaire, lundi 15 avril 2013

"À tout le monde

Arrêtez de penser qu'on est bêtes parce qu'on est en Clis.

On est intelligents, on est capables de travailler tout seuls.

On a juste des problèmes et la Clis nous aide et nous permet d'évoluer. 
On a notre place dans l'école, comme vous. "

Alec, Asim, Djamel, Éric, Riad, Romane, Tom.

Ces différentes dimensions de la liminalité opèrent donc dans un mouvement complexe parce qu'elles se contredisent parfois. Ainsi, les élèves qui ne connaissent pas de temps de scolarisation en classe ordinaire se trouvent placés dans la situation la plus liminale socialement, parce qu'ils sont les plus isolés du reste de la population ordinaire. Mais ce sont aussi ceux qui l'éprouvent le moins.

La réflexion collective des élèves amène à considérer la liminalité comme bénéfique parce que c'est bien la Clis 1 qui les autorise à revendiquer la normalisation exigée par l'institution. L'empan liminal permet de sortir du discours exclusivement dramatique de la liminalité, en en montrant les effets protecteurs et positifs.

\section{Une liminalité bénéfique}

Murphy parle du naufrage de l'identité de l'invalide due à sa situation liminale exclusivement dramatique, d'une part parce qu'elle est pérenne, d'autre part parce qu'elle n'a pas de fonction pédagogique. L'altération définitive du corps définit la mise à l'écart de l'invalide de la société, dont il n'est plus considéré comme un membre à part entière. Toutes les enseignantes soulignent que leur principal souci est d'abord de restaurer une image de soi des élèves, image flétrie par leurs années passées en classe ordinaire et les grandes difficultés auxquelles ils y ont été confrontés. C'est par cette première étape de leur travail qu'elles vont ensuite pouvoir faire accéder les élèves aux apprentissages proprement dits. La liminalité de ces enfants est un temps de transformation perpétuelle. La Clis 1 peut être considérée comme un lieu rééducatif, qui propose à l'enfant en situation de handicap de passer de l'état de l'élève en échec scolaire à celui d'un élève répondant aux normes scolaires. Ici, elle constitue bien le lieu d'une initiation par ce que l'on pourrait nommer une rééducation pédagogique.

Tous les élèves reconnaissent que la Clis 1 est une classe aidante: c'est grâce à elle qu'ils progressent scolairement. En dépit des contradictions exprimées, ils sont satisfaits d'avoir deux places dans l'école. Ils ont conscience de leurs difficultés et reconnaissent qu'une scolarisation à temps plein en classe ordinaire ne leur permettrait pas de progresser scolairement de la même façon.

Clis Baudelaire, 6 ${ }^{\mathrm{e}}$ séance de réflexion collective, lundi 18 mars 2013

Pourquoi tu préfèrerais rester en Clis, si tu n'aimes pas?

Éric: "Oui, parce que ça m'aide, c'est plus facile. "

C'est par rapport au travail que tu préfèrerais rester en Clis, plutôt que d'aller en classe d'inclusion?

Éric: "Oui." "

[...]

Est-ce que tu penses que si tu étais tout le temps en classe d'inclusion, tu arriverais à travailler?

Éric: "Ben, non."

Au fil des années passées en Clis et de l'augmentation progressive de temps de scolarisation en classe ordinaire, les élèves cessent de voir la Clis comme un lieu d'apprentissages. 


\section{Clis Balzac, $5^{\mathrm{e}}$ séance de réflexion collective, mardi 19 mars 2013}

Loman: "Moi, j'aime bien. Ben des fois, ici, dans la Clis, on nous aide à lire et tout ça, et dans l'autre classe, on fait des trucs sérieux. "

Plus sérieux que dans la Clis?

Loman: "Oui."

Qu'est-ce que tu entends par "sérieux"?

Loman: "Ben, plus dur. "

Anis: "Ben, je suis d'accord de Loman, ce qu'il dit. Parce qu'ici, des fois, ça me gêne trop, parce qu'il y a trop de travail, après, je suis fatigué. "

Il est notable que cette considération de la Clis se fait sur sa dévalorisation. La classe spécialisée est importante, parce que le travail n'y est pas sérieux, trop facile. Cette dévalorisation témoigne paradoxalement de la réussite de la Clis, par une reconnaissance implicite du travail qui s'y fait, puisque c'est effectivement grâce à celui-ci que les élèves ont pu être intégrés dans une classe ordinaire. Même s'ils dévalorisent la Clis au fil de leurs années scolaires, les élèves manifestent aussi leur reconnaissance envers une structure qui, malgré leurs difficultés d'apprentissages, leur octroie une possibilité de se maintenir dans un tissu social ordinaire. C'est pourquoi ils se sentent semblables aux autres, résistent à leur stigmatisation, ne se conforment pas à cette identité de handicapé définie par leur inscription en Clis 1 . On peut penser que, scolarisés à plein-temps dans une classe ordinaire, ces élèves auraient beaucoup plus de difficultés à se reconnaître autorisés à être dans ce lieu, parce qu'alors seules leurs difficultés seraient visibles et qu'ils seraient isolés du groupe. La Clis 1, par le sas qu'elle procure et l'aide effective qu'elle leur apporte, la solidarité du collectif, leur permet de s'inscrire dans les apprentissages, d'acquérir un statut d'élève du fait de leurs progrès reconnus au sein de la classe. Elle légitime leur présence dans l'école et leur permet d'affronter les autres. La liminalité a des effets positifs et n'est pas condamnée par ceux qui la vivent au quotidien.

\section{Une liminalité pérenne}

II n'en reste pas moins vrai que le retour définitif en classe ordinaire reste exceptionnel. Sur les vingt-neuf élèves avec lesquels j'ai mené ma recherche, une seule en est actuellement sortie, en raison de l'affirmation constante de son refus de sa présence en Clis, lors des séances de réflexion collective. Sa situation était cependant exceptionnelle: arrivée en Clis cette année, à l'âge de onze ans, orientée l'année suivante en Segpa ${ }^{3}$, Charlène ne correspondait pas au profil des autres élèves de la classe et a finalement intégré une classe de CM2 au mois de mars. Deux élèves, que je n'ai pas rencontrés, sont scolarisés à plein-temps en ordinaire, mais restent administrativement inscrits en Clis. Une enseignante envisage une sortie de Clis pour l'un d'eux, avec pour objectif une orientation en Segpa. Ainsi, sur tous ces élèves, un seul connaîtra une scolarité en 6e ordinaire. Certains autres quitteront le champ du handicap par une orientation en Segpa, mais celle-ci les maintiendra dans

3. Segpa: Section d'enseignement général professionnel adapté, accueillant de la $6^{e}$ à la $3^{e}$ des élèves "présentant des difficultés scolaires graves et durables " dans des classes séparées des autres classes du collège. 
une scolarité où ils resteront identifiés par leurs difficultés. La majorité d'entre eux resteront dans le champ du handicap, par une orientation en IME ou en Ulis collège ${ }^{4}$. Si la liminalité scolaire s'inscrit dans un processus dynamique, elle n'en constitue pas moins une situation pérenne pour la majorité de ces élèves.

\section{CONCLUSION}

Le concept de Murphy, repris de façon paradigmatique par les chercheurs français présente les personnes en situation de handicap comme des victimes impuissantes de la société, rejetées par les valides. La liminalité les réifie sans qu'elles ne puissent s'affirmer et participer pleinement à la vie sociale, prises dans une tension entre la société qui veut les renvoyer dans une communauté homogène qu'elles refusent et leur demande de reconnaissance entière dans cette société. La liminalité des personnes en situation de handicap constitue un drame définitif auquel elles ne peuvent pas échapper.

La recherche menée avec des élèves de Clis 1 montre une autre réalité. Si la Clis est bien le lieu de la liminalité au sein de l'espace scolaire, les élèves se défendent de toute réification affirmant avec force la validité de leur place à l'école et le rejet de toute différence: leurs difficultés reconnues ne suffisent pas à les distinguer identitairement des autres élèves. La liminalité n'est pas considérée de façon dramatique par les élèves, mais bien de façon bénéfique. C'est la Clis 1 qui autorise leur maintien dans le système scolaire dans des conditions plus ou moins satisfaisantes pour eux. En effet les perceptions individuelles des élèves varient selon leurs temps de scolarisation en classe ordinaire, faisant apparaître des situations liminales déclinées dans un empan.

L'empan liminal se définit donc comme un processus dynamique, évolutif au fil des années scolaires, caractérisant les différentes situations des personnes en situation de handicap, et comprenant différentes dimensions qui ne se recoupent pas toujours. La liminalité de la Clis 1 opère dans un mouvement complexe, parce qu'il relève de décisions institutionnelles mais aussi de perceptions individuelles qui contredisent parfois le fait social.

L'empan liminal de la situation scolaire des élèves de Clis 1 questionne alors la politique de l'éducation inclusive prônée par les textes ministériels. La logique inclusive exige un changement radical de l'institution scolaire (Kron, Plaisance, 2012) : ce n'est plus à l'enfant de s'adapter à son environnement, mais à celui-ci de s'adapter à l'enfant. La recherche met en avant des pratiques locales et diversifiées qui maintiennent cependant la logique intégrative dans l'école élémentaire. Le discours des enfants, corroboré par mes observations, montre que la centration sur la mission de transmission des savoirs occulte celle de la socialisation et empêche la mise en place d'une véritable éducation à la citoyenneté et du vivre ensemble qui constituent les éléments essentiels du concept d'inclusion.

4. Ulis collège: Unité localisée pour l'inclusion scolaire-collège (Ulis collège), dispositif collectif de scolarisation correspondant, dans le second degré, à celui de I'Ulis école. II existe également des Ulis lycée (circulaire 2015-129 du 21 août 2015, succédant à la circulaire 2010-088 du 18 juin 2010 sur les Ulis dans le second degré). 
L'empan liminal peut alors constituer un outil opératoire pour penser la question de l'inclusion et la situation de toute personne placée en marge de la société.

\section{Références}

Blanc, A. (2006). Le handicap ou le désordre des apparences. Paris: Armand Colin. Calvez, M. (1994). Le handicap comme situation de seuil: éléments pour une sociologie de la liminalité. Sciences sociales et santé, 12(1), 61-88.

Circulaire $n^{\circ} 2009-087$ du 17 juillet 2009. Scolarisation des élèves handicapés à l'école primaire; actualisation de l'organisation des Classes pour l'inclusion scolaire (Clis).

Delalande, J. (2001). La cour de récréation, Pour une anthropologie de l'enfance. Rennes: PUR.

Fablet, D. (2004). Pour d'autres modalités de collaboration entre chercheurs et professionnels de l'intervention socio-éducative: les apports d'approches socio-cliniques. Éduquer, 8, 43-53.

Freire, P. (2013). Pédagogie de I'autonomie. Toulouse: Érès. (1're édition: 2006). Gardou, Ch. (2009). Fragments sur le handicap et la vulnérabilité. Toulouse: Érès. (1 $1^{\text {re }}$ édition: 2005).

Kron, M., Plaisance, É. (2012). Grandir ensemble, l'éducation inclusive dès la petite enfance. Suresnes: INS HEA.

Lourau, R. (1970). L'analyse institutionnelle. Paris: Éditions de minuit.

Monceau, G. (2003). Pratiques socianalytiques et socio-clinique institutionnelle. L'homme et la société 1, 147, 11-33.

Monceau, G. (2005). Transformer les pratiques pour les connaître: recherche-action et professionnalisation enseignante. Educacão e Pesquisa, 31(3). São Paulo, Brésil. http://www.scielo.br/pdf/ep/v31n3/fr_a10v31n3.pdf

Monceau G. (dir.) (2012). L'analyse institutionnelle des pratiques Une socio-clinique des tourments institutionnels au Brésil et en France. Paris: L'Harmattan.

Murphy, R. F. (1990). Vivre à corps perdu: le témoignage et le combat d'un

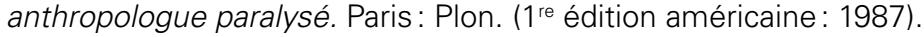

Sirota, R. (2006a). Petit objet insolite ou champ constitué, la sociologie de l'enfance est-elle dans les choux? In R. Sirota, R. (dir.), Éléments pour une sociologie de l'enfance (pp.13-34). Rennes: PUR.

Stiker, H.-J. (2005). Corps infirmes et sociétés. Paris: Dunod. (1 ${ }^{\text {re }}$ édition: 1982). Tisdall E. K. M., Davis J. M. \& Callagher M. (2009). Researching with Children \& Young People. London: SAGE Publications Ltd. P.

Turner, V. (1990). Le Phénomène rituel. Structure et contre structure. Paris: PUF. (1 ${ }^{\text {re }}$ édition américaine: 1969 ).

Van Gennep, A. (2001). Les rites de passage. Paris: Picard. (1're édition: 1909). 